\title{
Prizes awarded by the IAMP
}

Robert Seiringer (IST Austria, Klosterneuburg, Austria)

The International Association of Mathematical Physics (IAMP) has awarded the 2018 Henri Poincaré Prize for mathematical physics to: Michael Aizenman, Princeton University; Percy Deift, New York University; and Giovanni Gallavotti, Università di Roma La Sapienza.
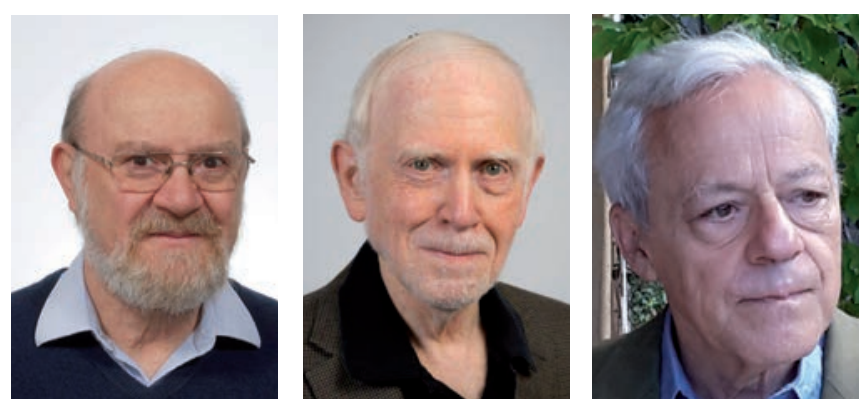

Michael Aizenman was honoured "for his seminal contributions to quantum field theory, statistical mechanics and disordered systems, in which he pioneered innovative techniques that demonstrate the beautiful and effective interplay between physical ideas, mathematical analysis, geometric concepts and probability theory". Percy Deift was honoured "for his seminal contributions to Schrödinger operators, inverse scattering theory, nonlinear waves, asymptotic analysis of Fredholm and Toeplitz determinants, universality in random matrix theory and his deep analysis of integrable models". Giovanni Gallovotti was honoured "for his outstanding contributions to equilibrium and non-equilibrium statistical mechanics, quantum field theory, classical mechanics and chaotic sys- tems, including, in particular, the renormalisation theory for interacting fermionic systems and the fluctuation relation for the large deviation functional of entropy production".

The Henri Poincaré Prize, which is sponsored by the Daniel Iagolnitzer Foundation, recognises outstanding contributions that lay the groundwork for novel developments in mathematical physics. It also recognises and supports young people of exceptional promise who have already made outstanding contributions to the field. The prize is awarded every three years at the International Congress on Mathematical Physics.

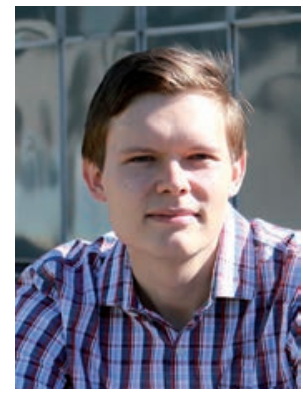

The IAMP has awarded the 2018 Early Career Award to Semyon Dyatlov (UC Berkeley/Massachusetts Institute of Technology) "for the introduction and the proof of the fractal uncertainty principle, which has important applications to quantum chaos and to observability and control of quantum systems". The Early Career Award is given in recognition of a single achievement in mathematical physics and is reserved for scientists aged under 35. It is sponsored by Springer.

The prizes were awarded on 23 July 2018 in Montreal, Canada. For prior winners, selection committee members and laudations, see http://www.iamp.org. 